\title{
Elastostatic modeling of multi-link flexible manipulator based on 2D dual-triangle tensegrity mechanism
}

\author{
Wanda Zhao \\ Laboratoire des Sciences du Numérique de Nantes (LS2N), UMR CNRS 6004 \\ 44321, Nantes, France \\ Wanda.Zhao@ec-nantes.fr
}

\section{Anatol Pashkevich \\ IMT Atlantique Bretagne Pays de la Loire \\ 44307, Nantes, France; \\ Anatol.Pashkevich@imt-atlantique.fr}

\author{
Alexandr Klimchik ${ }^{1}$ \\ Innopolis University, \\ Universitetskaya St, 1, Innopolis, Tatarstan, 420500, Russia \\ A.Klimchik@innopolis.ru
}

\section{Damien Chablat}

Centre National de la Recherche Scientifique (CNRS)

44321, Nantes, France ;

Damien.Chablat@cnrs.fr

\begin{abstract}
The paper deals with the elastostatic modeling of a multi-link flexible manipulator based on the $2 D$ dualtriangle tensegrity mechanism and its non-linear behavior under external loading. The main attention is paid to the static equilibriums and the manipulator stiffness behavior under the loading for the arbitrary initial configuration. It was proved that there is a quasi-buckling phenomenon for this manipulator while the external loading is increasing. In the neighborhood of these configurations, the manipulator behavior was analyzed using the enhanced Virtual Joint Method (VJM). A relevant simulation study confirmed the obtained theoretical results.

Keywords: Compliant manipulator; Tensegrity mechanism; Stiffness analysis; Robot buckling
\end{abstract}




\section{INTRODUCTION}

Compliant manipulators are used nowadays in many fields since their flexibility, modularized construction, and low weight. Many new mechanical structures were studied in this area [1-6], which showed quite good performances compared with traditional rigid robots. Recently, in literature, particular attention is paid to tensegrity mechanisms, which are made up of a series of similar segments composed of compressive and tensile elements (cables or springs) $[7,8]$. Such structures found their applications in space exploration robots $[9,10]$, in the design of new types of aerial vehicle [11] and manipulators [12], fish robot design [13], in the ocean wave energy harvesting mechanisms [14], design of new type of actuators [15] and twisting wing mechanisms [16]. One of such tensegrity structures is studied in this paper.

Tensegrity mechanisms belong to the class of highly over-constrained mechanisms where topological synthesis plays an important role. In order to find tensegrity structures that better suit the desired application different optimization methods were applied. For example, Chen et al. used ant colony optimization for tensegrity structure optimization [17]. Ehara and Kanno used mixed integer programming to minimize the number of cables and remove redundant self-equilibrium modes [18]. Koohestani used genetic algorithms for the form-finding of tensegrity structures based on force density optimization [19]. Wang et al. proposed a computational framework for the form-finding of tensegrity based on the rank minimization of force density matrix [20]. It should be noted that in some cases, the complexity of the tensegrity structures is not a barrier for analytical solutions for form- 
finding [21]. An improved symmetry method for analytical form-finding of tensegrity structures based on group representation theory and the force density method is proposed in [22]. The proposed approach can obtain all feasible stable or superstable tensegrity structures with expected symmetries analytically. Modeling and control of tensegrity structures are highly non-linear problems, which usually have multiple solutions. Many research groups dealing with designing an appropriate model and control $[23,24]$, using deep reinforcement learning $[25,26]$, model predictive control [27], and convex optimization [28]. Some researchers design models with negative stiffness components [29]; that idea is a promising approach for system property optimization. Another attractive property of tensegrity structures is the presence of multiple equilibria and the ability to switch between them in order to reach higher power capabilities [30]. Such an approach was efficiently applied by Melancon et. al. [31] to design multistable inflatable origami deployable structures at the metre scale. A similar approach was used to design a protective system for robotic rotorcraft based on the origami structures [32]. It should be mentioned that origami-based design and optimization of tensegrity structueres if is one of the most promessing approach widely used by the community $[33,34]$.

The above-presented analysis concludes that non-linear stiffness analysis plays an essential role for tensegrity structures and, together with multiple equilibrium configurations, gives the colossal potential for property enhancement and utilization in new areas. However, that requires the development of an appropriate mathematical tool, which will help us to analyze tensegrity structure behavior under different loads. 
Some aspects of the stiffness modeling using matrix structural analysis $[35,36]$ and virtual joint modeling [37-40], as well as stability analysis [41, 42], can be used from classical manipulator stiffness analysis. However, they require essential advancements to take into account all tensegrity structures particularities.

Stiffness properties of some tensegrity mechanisms have already been studied carefully. In [43], the authors considered the mechanism composed of two springs and two length-changeable bars. They analyzed the mechanism stiffness using the energy method, demonstrated that the mechanism stiffness might decrease under external loading with the actuators locked, which may lead to the "buckling" phenomenon. Also, in [44], the cable-driven X-shape tensegrity structures were considered; here, the authors investigated the influence of cable lengths on the mechanism equilibrium configurations, which may be both stable and unstable. The relevant analysis of the equilibrium configurations as well as the stability and singularity study can be found in [45]. Using these results, the authors obtained conditions under which the mechanism can work continuously, without the "buckling" or "jump" phenomenon. There is also some research studying a four-legged parallel platform [46], which is based on the compliant tensegrity mechanisms. The authors investigated the loaded equilibrium configurations and numerically computed the platform stiffness.

For robotics, similar to classical mechanics dealing with the Euler column, the buckling phenomenon can be detected, which is not typical and was rarely studied before $[37,38]$. It should be noted that in contrast to column "buckling of the element," here "geometrical buckling" of structure arises, which induced by much lower external 
loads. It is usually treated as an undesirable phenomenon because the robot may suddenly change its shape when the loading force exceeds some critical value. Engineers prefer to avoid buckling while designing a robot. However, such property can be helpful in some fields [47-49]. Also, sometimes the quasi-buckling phenomenon may occur, which changes the robot resistance in one direction suddenly while the external loading is increasing. It is not typical for robotics and was rarely studied before. For this reason, this phenomenon should be obligatory taken into account in stiffness analysis.

This paper is an extension of our previous results $[50,51]$, which concentrated on the stiffness analysis of the multi-link flexible manipulator based on the 2D dualtriangle tensegrity mechanism. It was assumed that each segment is a composition of two rigid triangle parts, which are connected by a passive joint in the center and two elastic edges on each side with controllable preload. In contrast to the previous results, here, we consider a general case with an arbitrary number of segments and its stiffness behavior under the loading.

\section{MECHANICS OF 2D DUAL-TRIANGLE TENSEGRITY MECHANISM}

Let us present first a single segment of the compliant serial manipulator under study. It consists of two identical rigid triangles connected by a passive joint whose rotation is constrained by two linear springs, as shown in Fig. 1. It is assumed that the mechanism geometry is described by two triangle parameters $(a, b)$, and the mechanism shape is defined by the central angle $q$, which is adjusted through two control inputs 
influencing the springs $L_{1}$ and $L_{2}$. Let us denote the spring lengths in the non-stress state as $L_{1}^{0}$ and $L_{2}^{0}$, and the spring stiffness coefficient as $k$.
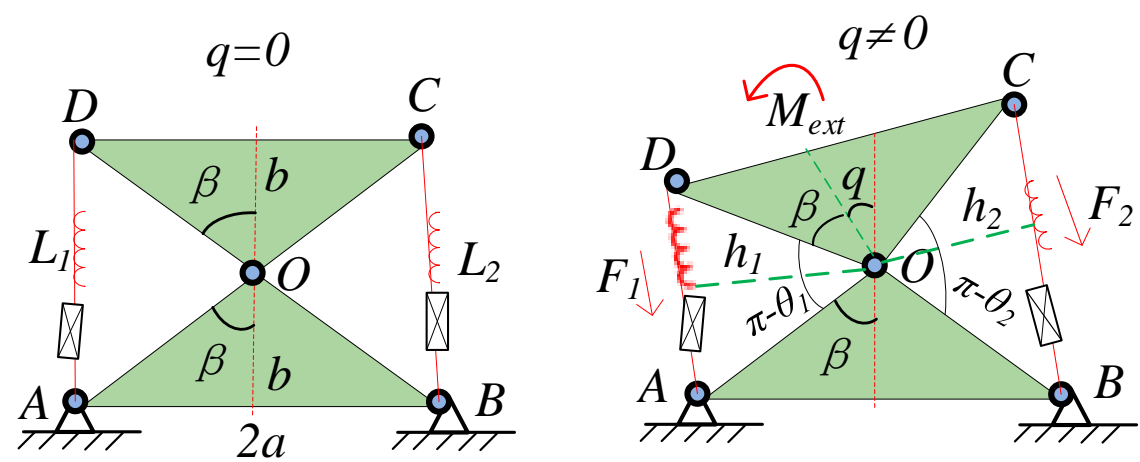

Fig. 1 Geometry of a dual-triangle mechanism

To find the mechanism configuration angle $q$ corresponding to the given control inputs $L_{1}^{0}$ and $L_{2}^{0}$, let us derive the static equilibrium equation of this mechanism, which can be easily computed using the elastic forces $F_{1}, F_{2}$ generated by the springs through Hook's law as

$$
F_{1}=k_{1}\left(L_{1}-L_{1}^{0}\right) ; \quad F_{2}=k_{2}\left(L_{2}-L_{2}^{0}\right)
$$

where the lengths $L_{1}$ and $L_{2}$ are the spring lengths $|A D|,|B C|$ corresponding to the current value of the angle $q$. These values can be computed from the triangles $\triangle A O D$ and $\triangle B O C$ using the formulas

$$
\begin{aligned}
& L_{1}\left(\theta_{1}\right)=c \cdot \sqrt{2+2 \cos \left(\theta_{1}\right)} \\
& L_{2}\left(\theta_{2}\right)=c \cdot \sqrt{2+2 \cos \left(\theta_{2}\right)}
\end{aligned}
$$


Where $c=\sqrt{a^{2}+b^{2}}$, and $\theta_{1}, \theta_{2}$ are expressed via the mechanism parameters $\theta_{1}=2 \beta+q, \theta_{2}=2 \beta-q$, and $\beta=\operatorname{atan}(a / b)$. That allows us to express the spring lengths $L_{1}\left(\theta_{1}\right)$ and $L_{2}\left(\theta_{2}\right)$ by the variable $q$ as $L_{1}(q)$ and $L_{2}(q)$ respectively. And the torques $M_{1}=F_{1} \cdot h_{1}, M_{2}=-F_{2} \cdot h_{2}$ generated by the springs in the passive joint $O$ can be computed using the triangle area relations $L_{1} h_{1}=c^{2} \sin \left(\theta_{1}\right), L_{2} h_{2}=c^{2} \sin \left(\theta_{2}\right)$ of $\triangle A O D$ and $\triangle B O C$, which gives us the following form.

$$
\begin{aligned}
& M_{1}(q)=+k\left(1-L_{1}^{0} / L_{1}(q)\right) c^{2} \sin (2 \beta+q) \\
& M_{2}(q)=-k\left(1-L_{2}^{0} / L_{2}(q)\right) c^{2} \sin (2 \beta-q)
\end{aligned}
$$

where $k$ denotes the springs stiffness coefficient, $L_{1}(q)$ and $L_{2}(q)$ are the current spring lengths, $L_{1}^{0}$ and $L_{2}^{0}$ are control inputs, while $c$ and $\beta$ are the geometric parameters described above (see Fig. 1). The difference in signs is caused by the different directions of the torques generated by the forces $F_{1} F_{2}$ with respect to the passive joint. So, taking into account, the external torque $M_{\text {ext }}$ applied to the moving platform, the static equilibrium equation for the considered mechanism can be written as

$$
M_{1}(q)+M_{2}(q)+M_{e x t}=0
$$


It is worth mentioning that both $M_{1}(q)$ and $M_{2}(q)$ highly depend on the spring initial prestress defined by the variables $L_{1}^{0}$ and $L_{2}^{0}$. Besides, the spring slackness is not possible here because of mechanical design particularities (i.e. springs are assumed to be able to generate both positive and negative efforts). It is also clear that this equation is highly nonlinear. Solving it numerically, we can get the rotation angle $q$ corresponding to the control inputs $L_{1}^{0}, L_{2}^{0}$ and the external torque $M_{\text {ext }}$ applied to the moving platform, and also obtain the torque-angle relation as follows

$$
M(q)=2 c k\left[c \cos \beta \sin q-L^{0} \cos (\beta / 2) \sin (q / 2)\right]
$$

where $M(q)=M_{1}(q)+M_{2}(q), L_{1}^{0}=L_{2}^{0}=L^{0}$.

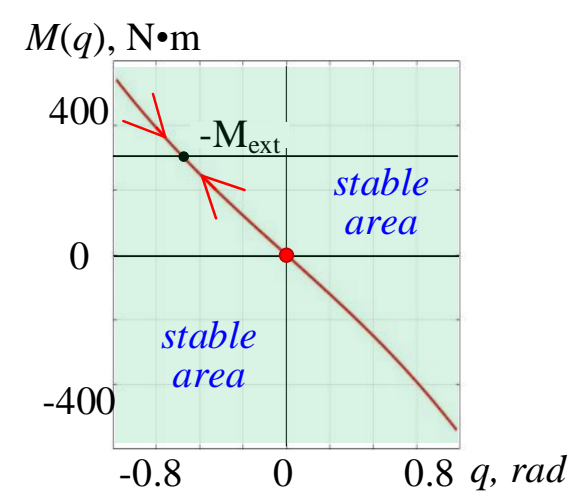

(a) monotonic case

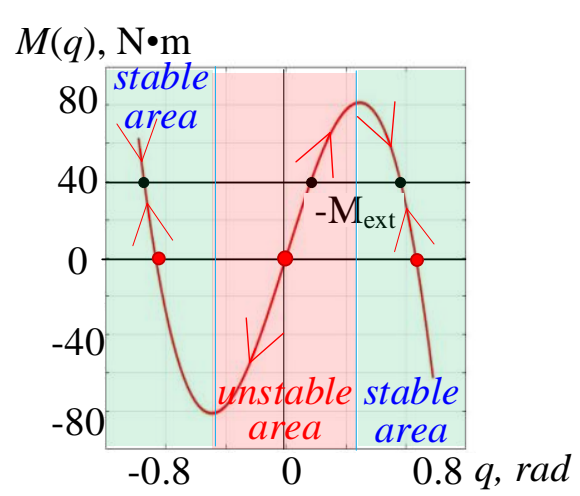

(b) non-monotonic case

Fig. 2 The torque-angle curves of dual-triangle mechanism 
It should be noted that this mechanism's static stability highly depends on the equilibrium configuration defined by $q$. As follows from the relevant analysis, the function $M(q)$ can be either a monotonic or non-monotonic one (Fig. 2), so the singlesegment mechanism may have multiple stable and unstable equilibriums, which are studied in detail in [50]. As follows from the relevant analysis, this mechanism's stability condition can be expressed via the derivative sign at the zero points, i.e. $\left.M^{\prime}(q)\right|_{q=0}<0$, which is easy to verify in practice. So, the relevant analytical expression for the derivative

$$
M^{\prime}(q)=c k\left[2 c \cos \beta \cos q-L^{0} \cos \frac{\beta}{2} \cos \frac{q}{2}\right]
$$

allows us to present the condition of the torque-angle curve monotonicity as follows

$$
L^{0}>2 b \cdot\left(1-(a / b)^{2}\right)
$$

This expression is extensively used below.

\section{STIFFNESS OF MULTI-LINK FLEXIBLE MANIPULATOR COMPOSED OF DUAL-TRIANGLES}

The serial manipulator considered in this paper is composed of $n$ similar sections connected in series, as shown in Fig. 3, where the left-hand side is assumed to be fixed. For the initial straight configuration, this manipulator's stiffness properties were studied 
in our previous paper, where the buckling phenomenon (similar to the Euler column) was discovered, and the critical force was computed. In this paper, a general case is considered where the manipulator's initial shape is assumed to be arbitrary, and the stiffness analysis is carried out for the loaded mode.

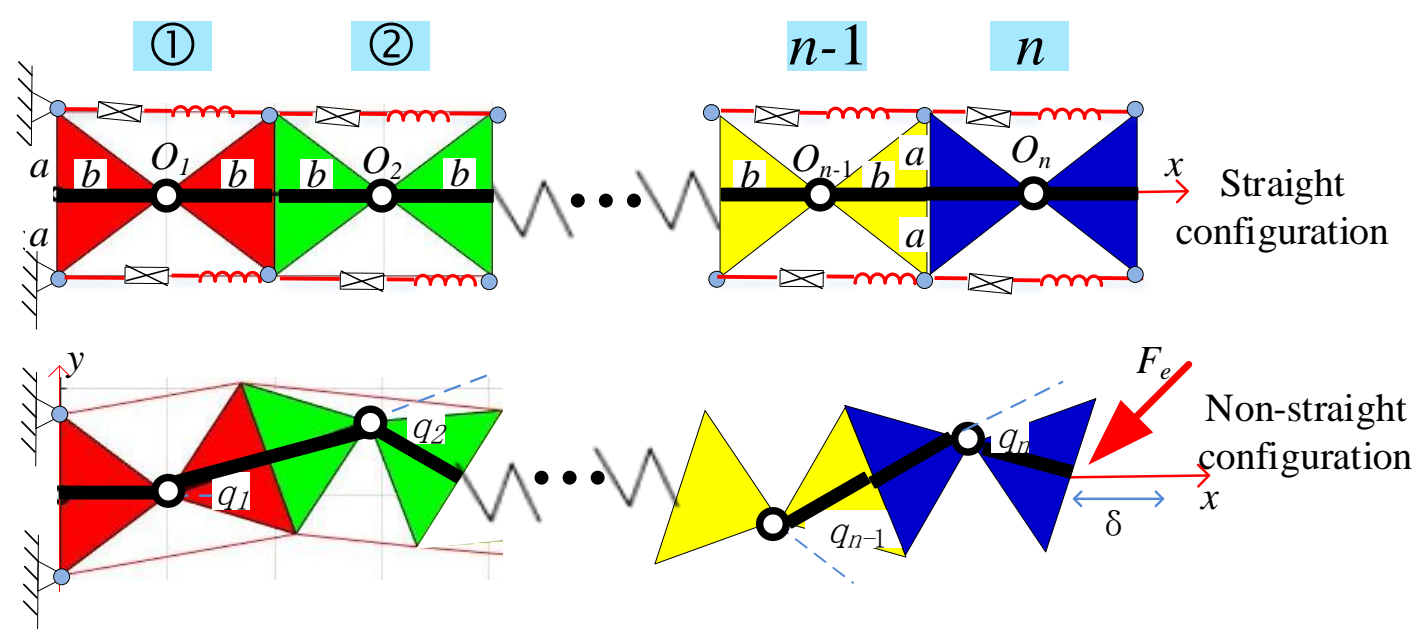

Fig. 3 Geometry of a multi-segment manipulator.

Let us assumed that the initial configuration of the $n$-link manipulator is a nonstraight one, which corresponds to the non-zero angles $\left(q_{i}^{0} \neq 0, i=1,2, \ldots, n\right)$ and the initial end-point location is $\left(x_{0}, y_{0}\right)=(2 n \cdot b-\Delta x, 0)$ with $\Delta x>0$. It is assumed that the corresponding control inputs $\left(L_{i 1}^{0}, L_{i 2}^{0}\right), i=1,2, . ., n$ are computed from the equilibrium conditions, where $L_{i 1}^{0}=L^{0}-\Delta_{i}, L_{i 2}^{0}=L^{0}+\Delta_{i}$ and $L^{0}=b$. It is clear that if $n \geq 3$ this manipulator is redundant with respect to the end-effector location control in the $(x, y)$ plane. So, for given $\left(x_{0}, y_{0}\right)$ the configuration angles $q_{i}^{0}$ cannot be computed uniquely. For this reason, we will consider two typical initial shapes of the manipulator, which in 
our previous paper were referred to as the U-shape and Z-shape [51]. Examples of such initial configurations for $n=4$ are presented in Table $1(\Delta x=0.3 b)$; their elastostatic properties will be carefully studied below.

Table 1 Two typical initial configurations of the manipulator for the end-point

$$
(x 0, y 0)=(7.7 b, 0)
$$

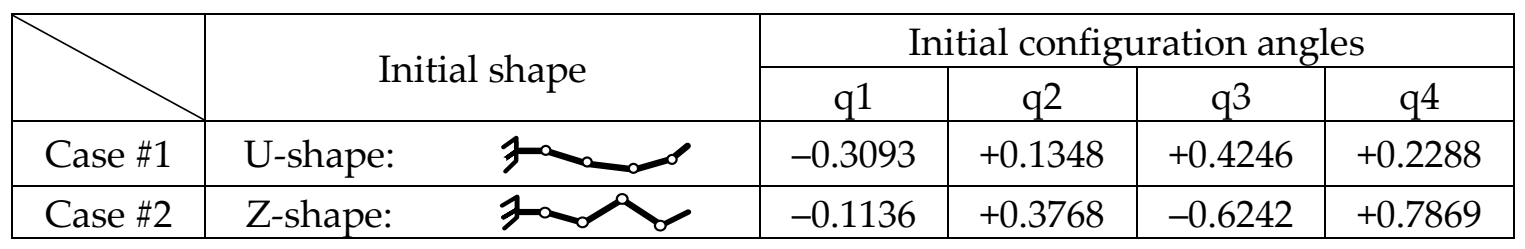

For any position the end-effector located in the workspace with the end-effector deflection $(\delta x, \delta y)$, the manipulator has its own equilibrium configurations and the corresponding external forces, so to have a better understanding of the force-deflection relations $F_{x}(\delta x)$ and $F_{y}(\delta x)$ corresponding to the end-effector displacement in the $(x$, y)-plane, let us assume the single constant control variable $\delta y=0$ and focus on $\delta x=$ var, i.e., from the initial location $\left(x_{0}, y_{0}\right)=(2 n \cdot b-\Delta x, 0)$ to the current one $(x, y)=(2 n \cdot b-\Delta x-\delta x, 0)$ where $\delta x$ is the end-effector deflection caused by the external forces $\left(F_{x}, F_{y}\right)$ and $\Delta x$ denotes the initial displacement of the end-effector. Let us apply the energy method allowing us to find possible equilibrium configurations corresponding to the given $\delta x$. It should be noted that the geometric constraint coming from the given end-effector location can be presented by the direct kinematic in the form 


$$
\begin{array}{r}
b+2 b \sum_{j=1}^{n-1}\left(\cos \sum_{i=1}^{j} q_{i}\right)+b \cos \sum_{i=1}^{n} q_{i}=2 n b-\Delta x-\delta_{x} \\
2 b \sum_{j=1}^{n-1}\left(\sin \sum_{i=1}^{j} q_{i}\right)+b \sin \sum_{i=1}^{n} q_{i}=0
\end{array}
$$

and allows us to reduce the number of variables in the energy function $E\left(q_{1}, q_{2}, \ldots q_{n-2}\right)$ by applying the 2-link manipulator inverse kinematics to compute the remaining angles $\left(q_{n-1}, q_{n}\right)$. Further, by detecting the $\mathrm{max} / \mathrm{min}$ and saddle points of the function $E\left(q_{1}, q_{2}, \ldots q_{n-2}\right)$, it is possible to find the configuration angles for all possible equilibriums. As known from the static analysis, the manipulator equilibriums must satisfy the following matrix equation.

$$
\mathbf{M}_{\mathbf{q}}+\left[\mathbf{J}_{\mathbf{q}}^{\mathrm{T}}\right]_{\mathbf{n} \times 2} \mathbf{F}=\mathbf{0}
$$

where $\mathbf{M}_{\mathbf{q}}=\left[M_{q 1}, M_{q 2}, \ldots, M_{q n},\right]^{T}$ is the matrix of the internal torques in manipulator joints generated by the springs forces, $\mathbf{F}=\left(F_{x}, F_{y}\right)^{T}$ is the external forces acting the manipulator end-effector, the Jacobian matrix $\mathbf{J}_{\mathbf{q}}$ of the manipulator can be expressed as

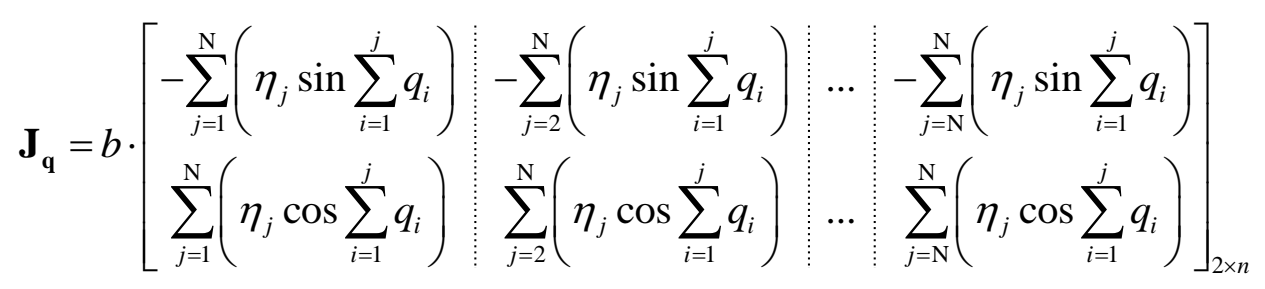


where $\eta_{j}=2$ for $j=1,2, \ldots, n-1$ and $\eta_{j}=1$ for $j=n$. To evaluate stability and compute the external forces $\left(F_{x}, F_{y}\right)$ corresponding to the end-effector deflection $\delta x$, let us apply the Moore-Penrose pseudo-inverse on the static equilibrium condition, which is shown as follows,

$$
\mathbf{F}=-\left[\mathbf{J}_{\mathbf{q}}^{\mathrm{T}}\right] \cdot\left[\mathbf{J}_{\mathbf{q}} \mathbf{J}_{\mathbf{q}}^{\mathrm{T}}\right]^{-1} \cdot \mathbf{M}_{\mathbf{q}}
$$

It should be mentioned that here both the Jacobian $\mathbf{J}_{\mathbf{q}}$ and the joint torques $\mathbf{M}_{\mathbf{q}}$ are computed using the configuration angles $q_{i}$ corresponding to the stable equilibriums.

Energy $E\left(q_{1}, q_{2}\right)$ for initial U-shape, $\delta x / b=0.4$
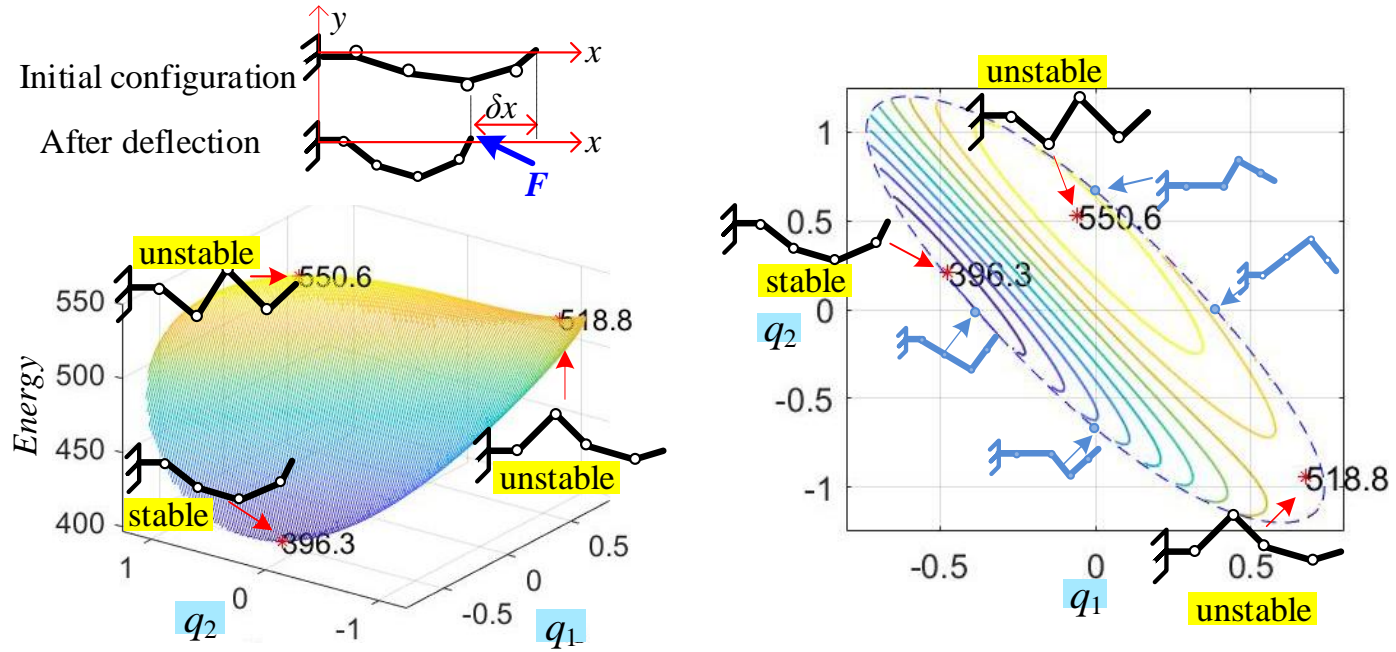

Fig. 4 The energy function and manipulator equilibriums for initial U-shape configuration (end-effector deflection $\delta x / b=0.4, \delta y=0$; geometric parameters $a / b=1.0 ; q 4>0)$.. 
Energy $E\left(q_{1}, q_{2}\right)$ for initial Z-shape, $\delta x / b=0.2$
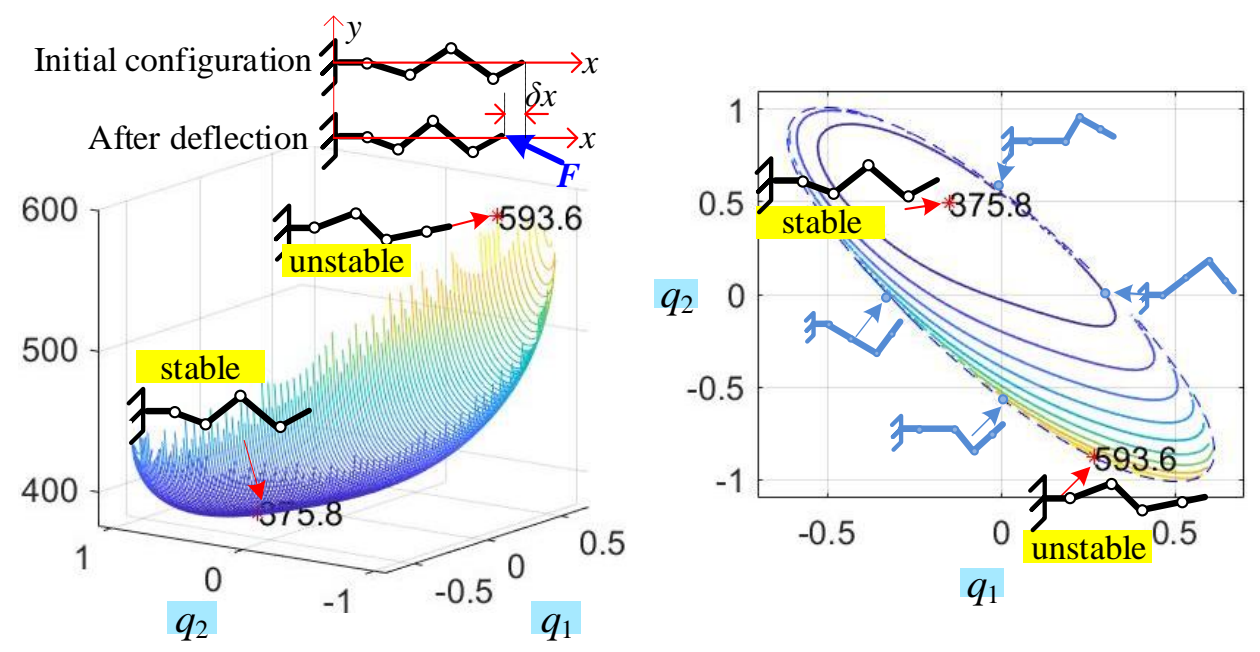

Fig. 5 The energy function and manipulator equilibriums for initial Z-shape configuration (end-effector deflection $\delta x / b=0.2, \quad \delta y=0 ; \quad$ geometric parameters $a / b=1.0 ; q 4>0)$.
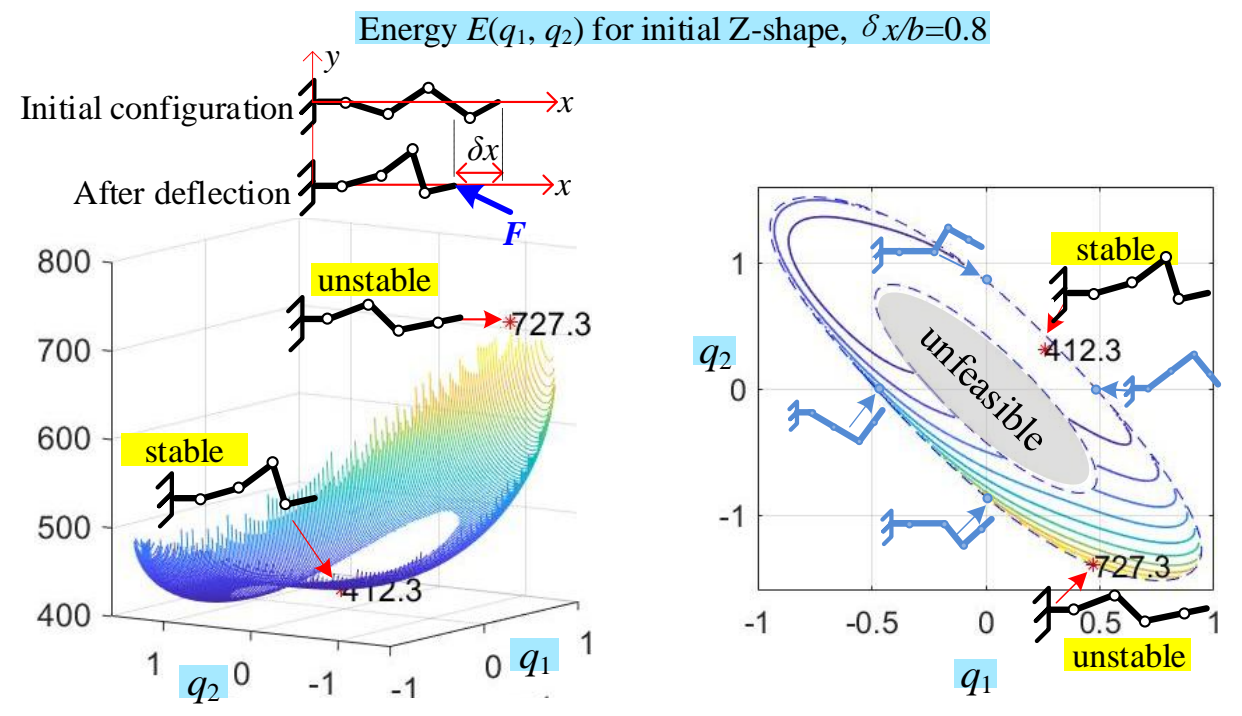

Fig. 6 The energy function and manipulator equilibriums for initial U-shape configuration (end-effector deflection $\delta x / b=0.8, \delta y=0$; geometric parameters $a / b=1.0 ; q 4>0)$. 
Examples of the obtain energy surfaces for $n=4$ are presented in Figs 4,5 and 6 , where the end-effector elastic deflection is $\delta x \in\{0.2 b, 0.4 b, 0.8 b\}$, and the initial shapes correspond to the end-effector displacement $\Delta x=0.3 b$ (see Table 1). As follows from these figures, for the initial U-shape (see Fig. 4), there are two cases of the energy surfaces $E\left(q_{1}, q_{2}\right)$ corresponding to $q_{4}>0$ and $q_{4}<0$, which are symmetrical. Totally, they have 6 critical points; each of them contains a single maximum, a single minimum and a single saddle point. Also, their evolution with respect to $\delta x$ is continuous; their topology remains the same while increasing the deflection $\delta x$. In contrast, for the initial Z-shape (see Figs. 5, 6), the energy surfaces $E\left(q_{1}, q_{2}\right)$ are quite different; their evolution with respect to $\delta x$ is discontinuous. The latter leads to sign-changing of some configuration angles $q_{i}$ under the external loading $F$ as shown in the figures (see angle $q_{1}$ for instance). Besides, if the deflection $\delta x$ is large enough, as in Fig. 6, the energy surfaces may contain a "hole," i.e., an unfeasible area caused by the violation of the geometric constraints $\left|q_{i}\right| \leq q_{i}^{\max }$ inside the manipulator segments.

\section{EVOLUTION OF MANIPULATOR STIFFNESS PROPERTIES UNDER THE LOADING}

By applying the above-presented energy method and computing minimums of the energy function $E\left(q_{1}, q_{2}, \ldots q_{n-2}\right) \rightarrow$ min for different $\delta x$, it is possible to obtain the desired force-deflection relations $F_{x}(\delta x)$ and $F_{y}(\delta x)$ describing the manipulator 
stiffness properties. Examples of such computations for $n=4$ are presented in Figs 7 and 8.

For the initial U-configuration (see Fig. 7), the change of the manipulator shape is smooth, the manipulator resistance against the external loading is gradually increasing while the deflection $\delta x$ becomes larger. Also, the stiffness coefficient in the $x$-direction is decreasing continuously. This tendency is observed until the manipulator reaches its geometric constraints. In contrast, for the initial Z-configuration (see Fig. 8), there are two intervals of the manipulator deformation. In the beginning, when $\delta x$ is relatively small, the manipulator maintains its Z-shape and the resistance against the external force is monotonically increasing, similar to the previous case.
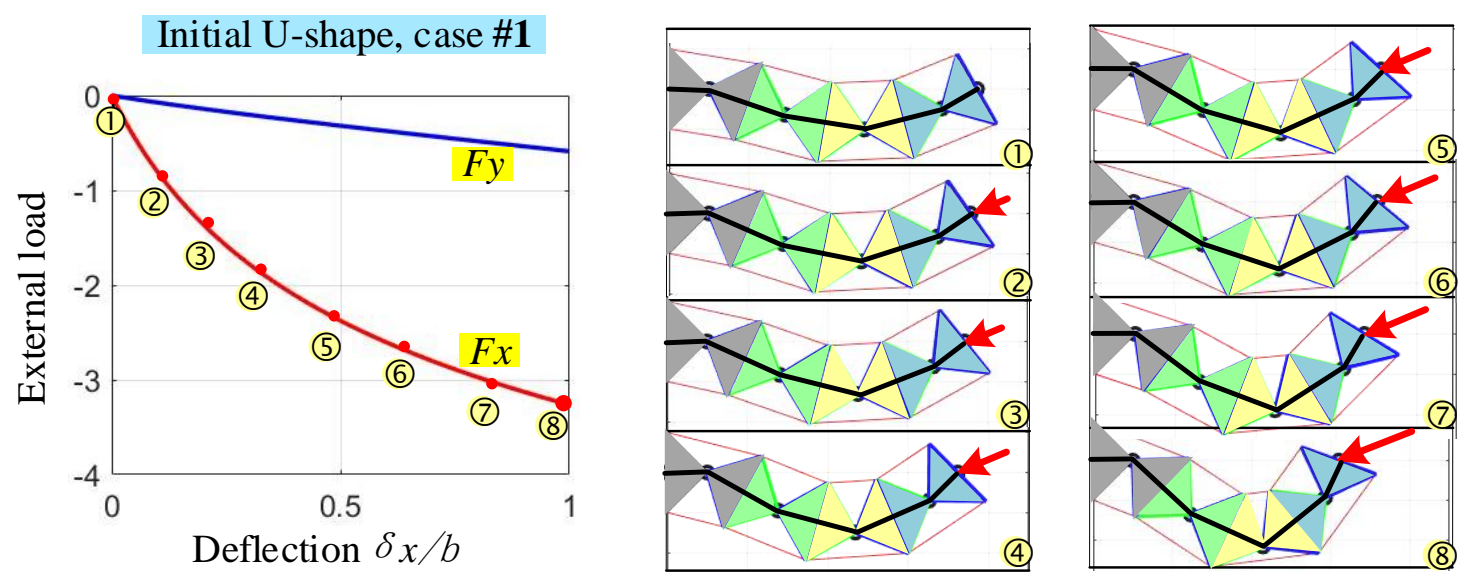

Fig. 7 Force-deflection curves $\mathrm{Fx}(\delta \mathrm{x}), \mathrm{Fy}(\delta \mathrm{x})$ and manipulator shape changing under the loading for initial U-shape for $(x 0, y 0)=(7.7 b, 0)$, geometric parameters $\mathrm{a} / \mathrm{b}=1.0$ and $\delta \mathrm{y}=0$. 

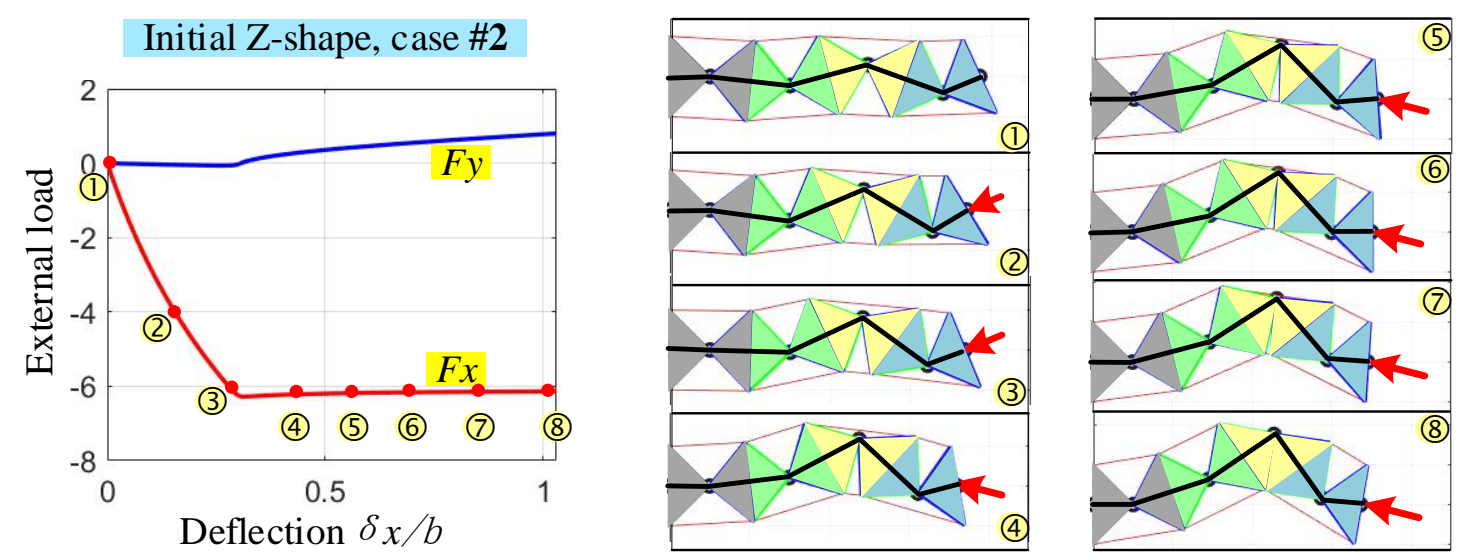

Fig. 8

Force-deflection curves $F x(\delta x)$, Fy $(\delta x)$ and manipulator shape changing under the loading for initial Z-shape for $(x 0, y 0)=(7.7 b, 0)$, geometric parameters $\mathrm{a} / \mathrm{b}=1.0$ and $\delta \mathrm{y}=0$.

Further, when the deflection $\delta x$ is larger than some critical value, the buckling phenomenon occurs, and the manipulator resistance against the external force is not increasing anymore. Correspondingly, the stiffness coefficient $d F_{x} / d x$ becomes very small, the stiffness coefficient $d F_{y} / d x$ changes its sign and the manipulator does not keep its initial Z-shape (some of the angles $q_{i}$ change the signs). Finally, after the buckling, the manipulator moves in the direction of its internal geometric constraints. Some more details concerning the evolution of the manipulator shape and its stiffness coefficients under the loading for both $x$ - and $y$-directions are presented in Table 2. 
Table 2 Evolution of the manipulator shape under the loading for $\delta x=v a r, \delta y=0$.

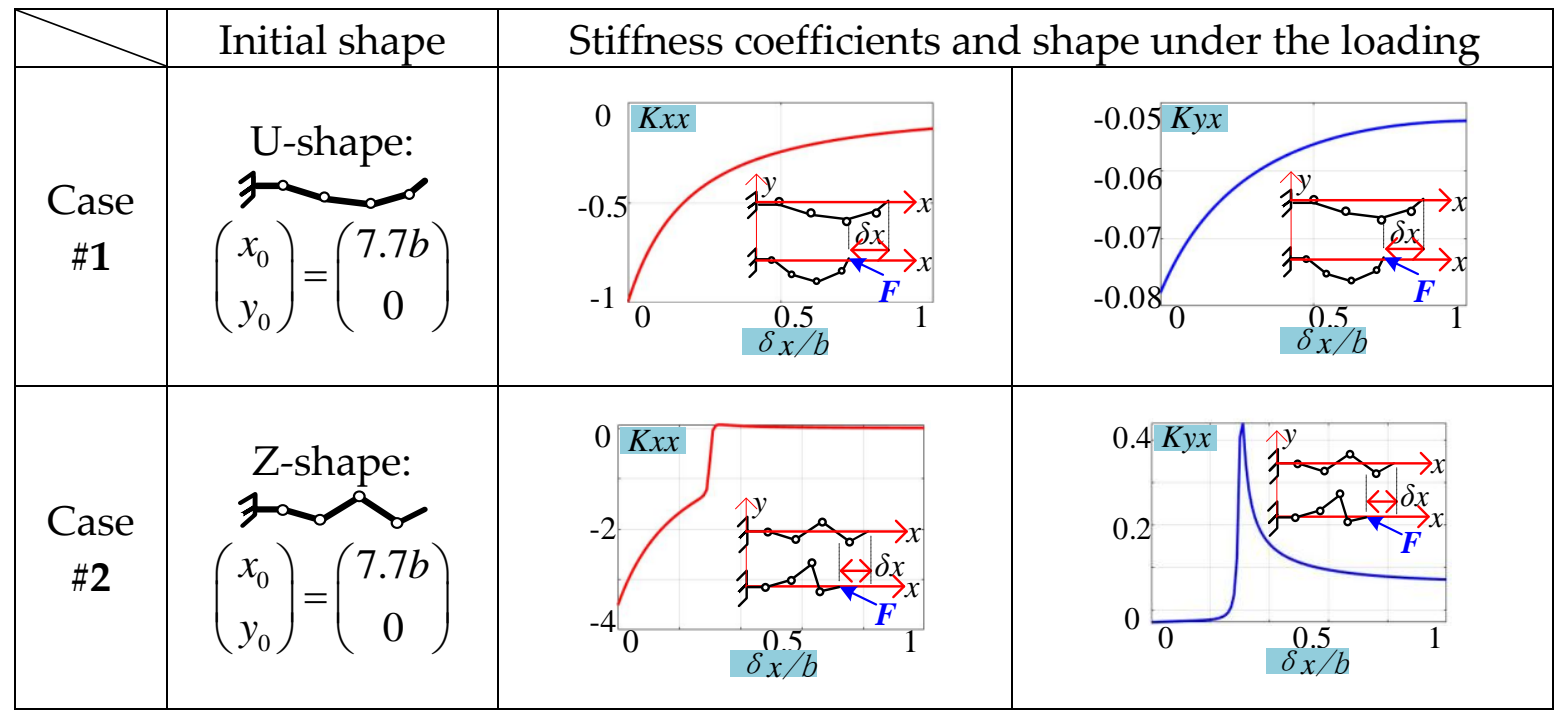

Hence, in practice, it is preferable to use the U-shape of the manipulator if the task space obstacles (external constraints) allow. It should also be noted that for the Zshape, it is necessary to avoid high loadings exceeding the critical force causing buckling.

Further, in addition to the above presented force-deflection relations $F_{x}(\delta x)$ and $F_{y}(\delta x)$ derived from the assumption of $\delta x=$ var, $\delta y=0$, let us analyze the changing of the manipulator stiffness coefficients under the loading $\left(F_{x}, F_{y}\right)$ without imposing any kinematic constraints of the end-effector location. To obtain the desired relations, it is necessary to compute the configuration angles $\left(q_{1}, \ldots, q_{n}\right)$ corresponding to the manipulator equilibriums for different given external forces $\left(F_{x}, F_{y}\right)$. It is clear that these angles can be found numerically by solving a system of $n$ independent equations (9) $\mathbf{M}_{\mathbf{q}}+\left[\mathbf{J}_{\mathbf{q}}^{\mathbf{T}}\right]_{\mathbf{n} \times \mathbf{2}} \mathbf{F}=\mathbf{0}$, which describes the static equilibrium condition (by applying Newton's method for instance). However, the initial guess of the angles 
$\left(q_{1}^{0}, \ldots, q_{n}^{0}\right)$ should be evaluated correctly to ensure that they are in the minimum energy configuration neighborhood because only such cases can be observed in practice. Such an initial guess can be obtained using the above-presented energy method applied in the space $\left(q_{1}, \ldots, q_{n}\right)$ with a rather rough grid with large step. Also, the desired angles corresponding to the external loading $\left(F_{x}, F_{y}\right)$ can be found using the Matlab function fminsearch, which minimizes the sum of the squared residuals i.e.

$$
\mathbf{q}=\arg \min _{\mathbf{q}}\left(\left\|\left[\mathbf{M}_{\mathbf{q}}\right]_{n \times 1}+\left[\mathbf{J}_{\mathbf{q}}^{\mathrm{T}}\right]_{n \times 2} \cdot \mathbf{F}\right\|^{2}\right)
$$

Where both the internal torques $\mathbf{M}_{\mathbf{q}}$ and the Jacobian $\mathbf{J}_{\mathbf{q}}$ depend on the angles $\mathbf{q}=\left(q_{1}, \ldots, q_{n}\right)^{T}$. It should also be mentioned that it is possible to simplify the problem of the initial guess $\left(q_{1}^{0}, \ldots, q_{n}^{0}\right)$ selection by gradually increasing the forces $\left(F_{x}, F_{y}\right)$ and using solutions from the previously loaded equilibrium as the initial guess for the next one corresponding to $\left(F_{x}+\Delta F_{x}, F_{y}+\Delta F_{y}\right)$. However, when the forces $\left(F_{x}, F_{y}\right)$ approach the buckling point, the initial guess from the previous step is not suitable because the configuration angles are changing essentially and only the straightforward energy method allows to obtain the correct initial guess.

If the equilibrium configuration angles $\left(q_{1}, \ldots, q_{n}\right)$ corresponding to the given force $\left(F_{x}, F_{y}\right)$ are computed, it is possible to find the desired stiffness coefficients using the formula for the loaded case [52], 


$$
\mathbf{C}_{\mathbf{F}}=\mathbf{J}_{\mathbf{q}}\left(\mathbf{K}_{\mathbf{q}}-\mathbf{K}_{\mathrm{g}}\right)^{-1} \mathbf{J}_{\mathbf{q}}^{\mathrm{T}} ; \quad \mathbf{K}_{\mathbf{F}}=\left[\mathbf{J}_{\mathbf{q}}\left(\mathbf{K}_{\mathbf{q}}-\mathbf{K}_{\mathrm{g}}\right)^{-1} \mathbf{J}_{\mathbf{q}}^{\mathrm{T}}\right]^{-1}
$$

where $\mathbf{K}_{\mathbf{F}}$ is the loaded stiffness matrix and $\mathbf{C}_{\mathbf{F}}$ is the corresponding compliance matrix. They include two essential components, the first of which $\mathbf{K}_{\mathbf{q}}$ corresponds to the unloaded case, and the second one $\mathbf{K}_{\mathrm{g}}$ describes the external force influence on the stiffness. In this expression, the $n \times n$ matrix of the joint elastic stiffness coefficients $\mathbf{K}_{\mathbf{q}}=\operatorname{diag}\left(K_{q 1}, \ldots, K_{q n}\right)$ can be computed using the derivative of the segment torque equation (5) as follows

$M(q)=2 c^{2} k \cos \beta_{12} \sin q-c k\left(L_{i 1}^{0} \sin \frac{\beta_{12}+q}{2}-L_{i 2}^{0} \sin \frac{\beta_{12}-q}{2}\right), i=1,2, \ldots, n$

where $L_{1}^{0} \neq L_{2}^{0}$. That yields the manipulator joint stiffness coefficient as

$$
K_{q i}=2 k\left(b^{2}-a^{2}\right) \cos q_{i}-k\left(\frac{L_{i 1}^{0}+L_{i 2}^{0}}{2} b \cdot \cos \frac{q_{i}}{2}-\frac{L_{i 1}^{0}-L_{i 2}^{0}}{2} a \cdot \sin \frac{q_{i}}{2}\right), i=1,2, \ldots, n
$$

It should be stressed that here, the control inputs $L_{i 1}^{0}$ and $L_{i 2}^{0}$ are constant values, which correspond to the initial unloaded joint angles $q_{i}$ that satisfying to the stable equilibrium for which $M(q)=0$. The second matrix $\mathbf{K}_{\mathrm{g}}$ containing the stiffness coefficients caused 
by the external loading is symmetrical and can be computed from the partial derivative of the Jacobian matrix as $\mathbf{K}_{\mathrm{g}} @ \partial \mathbf{J}^{\mathrm{T}} / \partial q_{i} \mathbf{F}$, which gives us

$$
\mathbf{K}_{\mathbf{g}}=\left[\begin{array}{c:c:c:c}
-J_{21} F_{x}+J_{11} F_{y} & -J_{22} F_{x}+J_{12} F_{y} & \ldots & -J_{2 n} F_{x}+J_{1 n} F_{y} \\
\ldots & -J_{22} F_{x}+J_{12} F_{y} & \ldots & -J_{2 n} F_{x}+J_{1 n} F_{y} \\
\ldots & \ldots & \ldots & \ldots \\
\ldots & \ldots & \ldots & -J_{2 n} F_{x}+J_{1 n} F_{y}
\end{array}\right]
$$

where $J_{i j}$ denotes the Jacobian matrix element $\mathbf{J}_{\mathbf{q}}$ from (10) corresponding to the $i$ th row and jth column.

It is evident that when the external forces are equal to zero, the stiffness matrix expression is reduced to the form, which is known from the unloaded mode analysis $\mathbf{K}_{0}=\left[\mathbf{J}_{\mathbf{q}} \mathbf{K}_{\mathbf{q}}^{-1} \mathbf{J}_{\mathbf{q}}^{\mathrm{T}}\right]^{-1}$. It should also be mentioned that, in contrast to the classical $n$-link serial manipulators, here the diagonal matrix $\mathbf{K}_{\mathbf{q}}$ is configuration dependent (not constant) because each initial configuration with the angles $\left(q_{1}, \ldots, q_{n}\right)$ produces its own control inputs $L_{i 1}^{0}$ and $L_{i 2}^{0}$ included in the expression (10). Besides, here the unloaded compliance matrix $\mathbf{C}_{0}$ can be expressed analytically in the following way

$$
\mathbf{C}_{0}=\left[\begin{array}{cc}
\frac{J_{11}^{2}}{K_{q 1}}+\frac{J_{12}^{2}}{K_{q 2}}+\ldots+\frac{J_{1 n}^{2}}{K_{q n}} & * \\
* & \frac{J_{21}^{2}}{K_{q 1}}+\frac{J_{22}^{2}}{K_{q 2}}+\ldots+\frac{J_{2 n}^{2}}{K_{q n}}
\end{array}\right]
$$


To illustrate the above-presented results' practical importance, they were applied to the case $n=4$, assuming that the initial (unloaded) endpoint location is $\left(x_{0}, y_{0}\right)=(7.7 b, 0)$, and the initial shape is either $\mathrm{U}$ - or $\mathrm{Z}$ - one. The configuration angles under the loading, corresponding to the external force $F=\left(F_{x}, F_{y}\right)$, were computed numerically using the proposed technique. Relevant results of the initial U-shape and Zshape are presented in Figs. 9 and 10, respectively. 
(a) $F x=v a r, F y=0$

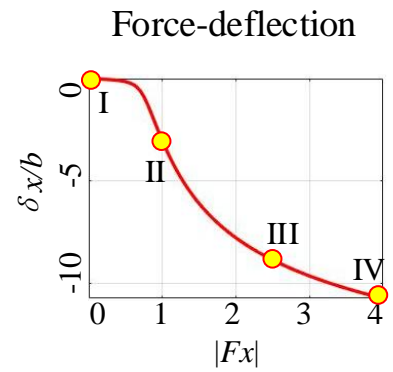

Stiffness coeff.
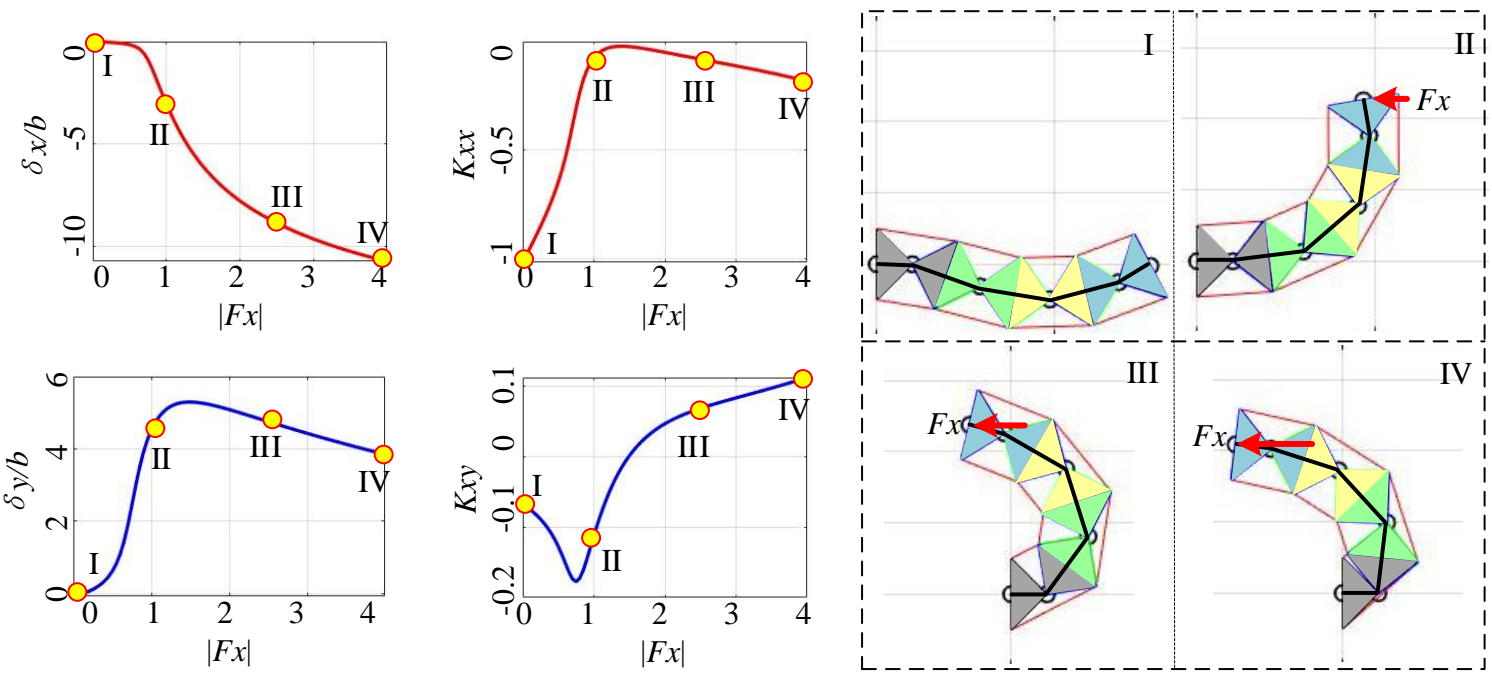

(b) $F x=0, \quad F y=v a r$

Force-deflection

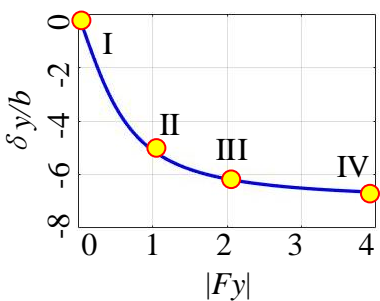

Stiffness coeff
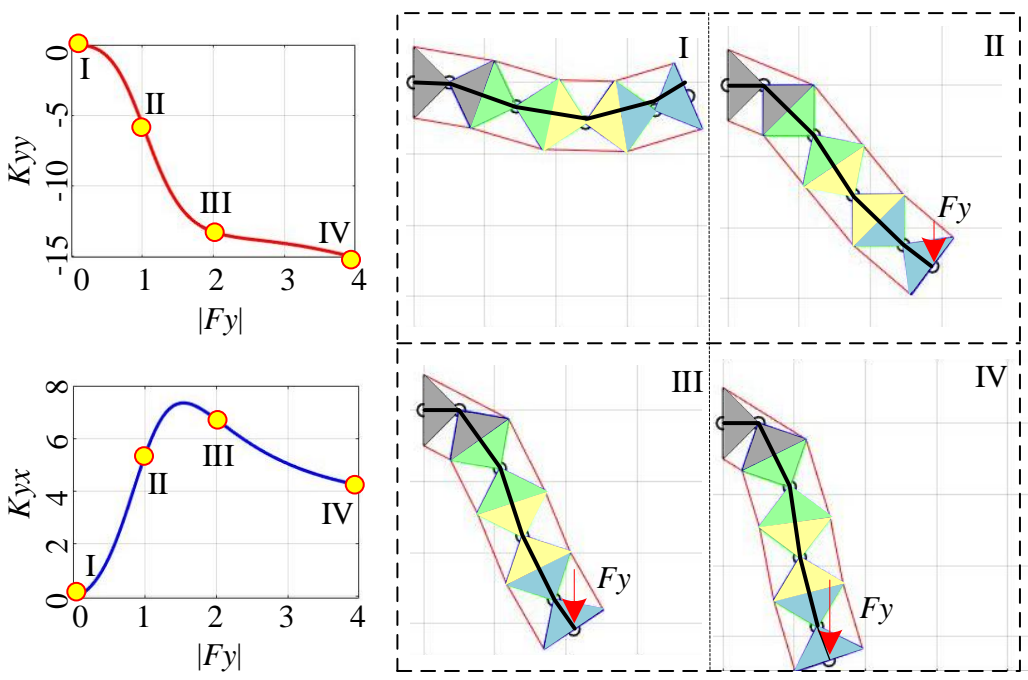

Fig. 9

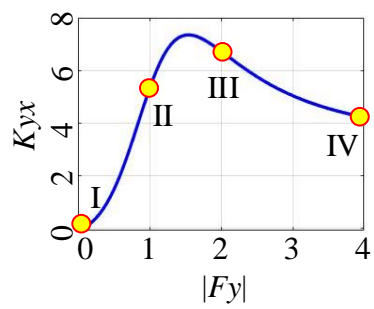

As follows from these figures, the manipulator stiffness essentially changes if the external loading is applied. For the initial U-shape case, the absolute value of the manipulator stiffness coefficient $\left|K_{\mathrm{xx}}\right|$ decreases first, while the force $F_{\mathrm{x}}$ is increasing 
(see Fig. 9a) until $F_{x}$ is reaching some critical value when $\left|K_{\mathrm{xx}}\right|$ is the minimum, then it begins to increase slowly. In contrast, the stiffness coefficient $K_{\mathrm{xy}}$ (describing the manipulator reaction in the $y$-direction) changes its sign under the loading. These stiffness properties can also be interpreted from the geometrical and physical point of view, using the right-hand side of Fig. 9a, which shows the evolution of the manipulator configuration under the loading. In general, such manipulator behavior can be treated as "quasi-buckling" because for certain loading $F_{\mathrm{x}}$ the stiffness in both $x$-and $y$-direction is very small. And the manipulator rotates quickly until one of the segments goes close to its joint limits, where the equivalent rotational stiffness coefficient is very low. Hence, in practice, it is necessary to avoid applying too high loading in $x$-direction causing approaching either to the "quasi-buckling" or the joint limits and losing the manipulator stiffness.

$$
F x=v a r, \quad F y=0
$$
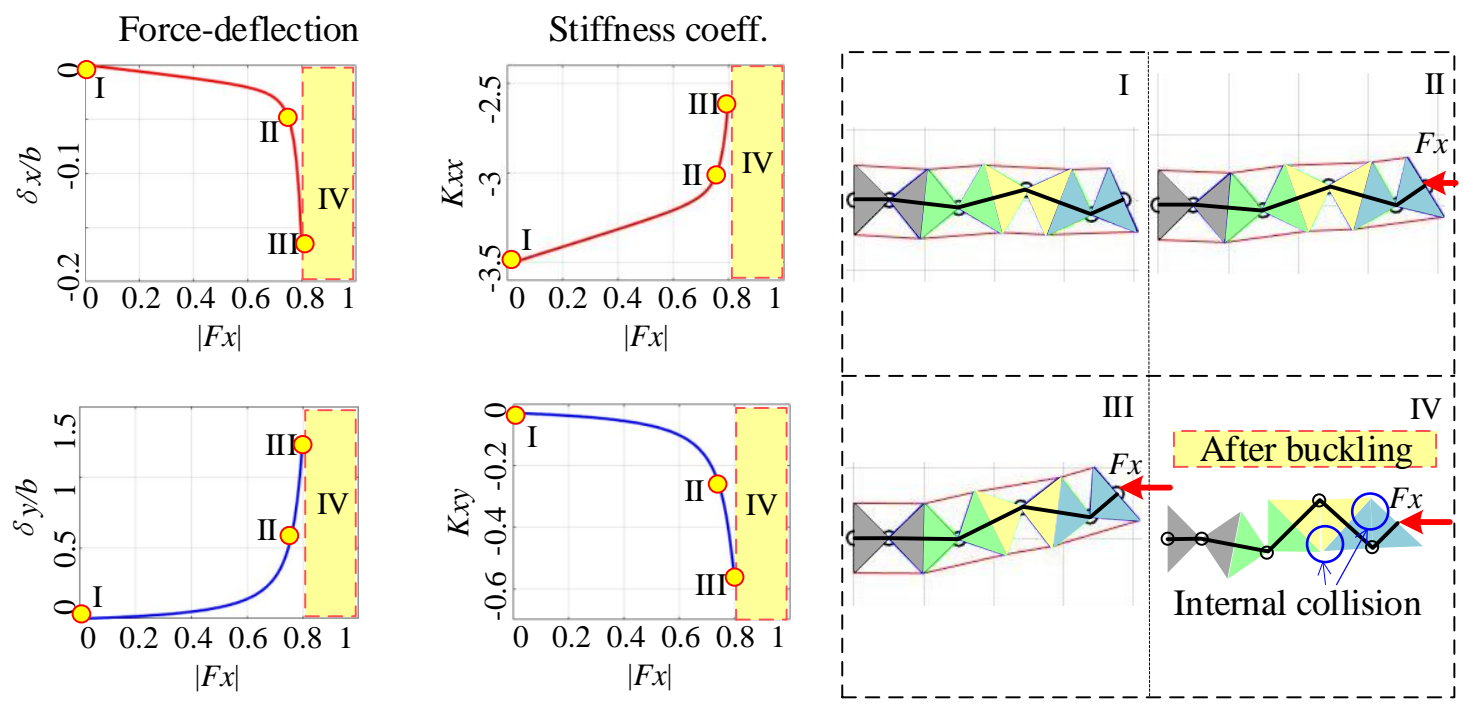

Fig. 10 Stiffness coefficients under the Fx-loading for initial Z-shape configuration with $(x 0, y 0)=(7.7 b, 0)$ and geometric parameters $a / b=1.0$ 
On the other side, while increasing the force $F_{\mathrm{y}}$ (i.e., in the orthogonal direction), the absolute value of the stiffness coefficient $\left|K_{\mathrm{yy}}\right|$ is monotonically increasing first, then it keeps the same tendency slowly (see Fig. 9b) because of the restriction of the geometric length of the manipulator. At the same time, the stiffness coefficient $K_{\mathrm{yx}}$ demonstrates non-monotonic behavior. Such performance can be seen from the evolution of the manipulator configuration at the right-hand side of Fig. 9b, where the manipulator end-point moves towards the extreme location, as far as possible from the initial one. Therefore, the high loading in $y$-direction should also be avoided to prevent the manipulator from changing its shape change to a pure straight line (see case IV).

However, for the second case study dealing with the initial Z-shape, the stiffness properties under the loading are quite different compared to the U-shape case. In particular, as follows from Fig. 10, under the $F_{x}$-loading, the absolute value of the stiffness coefficient $\left|K_{\mathrm{xx}}\right|$ decreases gradually at the beginning, then it decreases quickly to zero. In contrast, the absolute value of the stiffness coefficient $\left|K_{\mathrm{xy}}\right|$ increases monotonically. This phenomenon can also be treated as "quasi-buckling" because for particular loading, the manipulator stiffness in the $x$-direction is equal to zero, and the stiffness in the $y$-direction is very high. These results are illustrated geometrically by the right-hand side of Fig. 10, showing the manipulator configuration's evolution under the $F_{\mathrm{x}}$ loading. It is clear that here each segment of the manipulator tends to move close to its geometric limits before the "quasi-buckling" is occurring. In this configuration, even a quite small change of the external force may lead to large manipulator deflection, so in 
practice, it is reasonable to avoid such situations. It is worth mentioning that the case of $F_{\mathrm{y}}$-loading is not presented in Fig. 10 because it is quite similar to the $\mathrm{U}$-shape case.

Hence, for the manipulator understudy, the stiffness properties are essentially non-linear with respect to the loading force. Moreover, if the loading exceeds a particular value, the stiffness coefficients may become very low or even change their sign. The latter may be treated as the quasi-buckling, which normally should be avoided.

\section{CONCLUSIONS}

The paper focuses on the elastostatic modeling of a multi-link flexible manipulator based on the 2D dual-triangle tensegrity mechanism and its non-linear behavior under external loading. It is a specific case of the tensegrity mechanisms that currently are widely used in soft robotics. The primary attention is paid to the initial non-straight configuration of the manipulator. It was proved that under the external loading there might be the quasi-buckling phenomenon, which suddenly changes the manipulator resistance in one direction of its deflection but may do not influence the resistance in another direction. It was also demonstrated that there are usually six equilibrium configurations of this manipulator (two stable ones and four unstable ones). But if the end-effector's deflection is large enough some of the equilibriums may be unfeasible due to the geometric constraints.

To find the possible equilibriums and to analyze the manipulator shape under the loading, the energy method was used. Further, the stiffness analysis was based on the VJM approach allowing to find linearized relations between the end-effector 
deflection and the external force. The relevant simulation confirmed the obtained results. In the future, this technique will be used for the development of relevant control algorisms and related redundancy resolution.

\section{ACKNOWLEDGMENT}

This work was supported by the China Scholarship Council (No. 201801810036) and a

grant from the Russian Science Foundation (project No:19-79-10246).

\section{REFERENCES}

[1] Y. Yang and W. Zhang, "An elephant-trunk manipulator with twisting flexional rods," in 2015 IEEE International Conference on Robotics and Biomimetics (ROBIO), 6-9 Dec. 2015 2015, pp. 13-18, doi: 10.1109/ROBIO.2015.7407012.

[2] L. L. Howell, "Compliant Mechanisms," London, 2013: Springer London, in 21st Century Kinematics, pp. 189-216.

[3] M. Rolf and J. J. Steil, "Constant curvature continuum kinematics as fast approximate model for the Bionic Handling Assistant," in 2012 IEEE/RSJ International Conference on Intelligent Robots and Systems, 7-12 Oct. 2012 2012, pp. 3440-3446, doi: 10.1109/IROS.2012.6385596.

[4] M. Y. Wang and S. Chen, "Compliant Mechanism Optimization: Analysis and Design with Intrinsic Characteristic Stiffness," Mechanics Based Design of Structures and Machines, vol. 37, no. 2, pp. 183-200, 2009/05/12 2009, doi: 10.1080/15397730902761932.

[5] A. Albu-Schaffer et al., "Soft robotics," IEEE Robotics \& Automation Magazine, vol. 15, no. 3, pp. 20-30, 2008, doi: 10.1109/MRA.2008.927979.

[6] M. I. Frecker, G. K. Ananthasuresh, S. Nishiwaki, N. Kikuchi, and S. Kota, "Topological Synthesis of Compliant Mechanisms Using Multi-Criteria Optimization," Journal of Mechanical Design, vol. 119, no. 2, pp. 238-245, 1997, doi: $10.1115 / 1.2826242$.

[7] R. E. Skelton and M. C. De Oliveira, Tensegrity systems. Springer, 2009.

[8] K. W. Moored, T. H. Kemp, N. E. Houle, and H. Bart-Smith, "Analytical predictions, optimization, and design of a tensegrity-based artificial pectoral fin," International Journal of Solids and Structures, vol. 48, no. 22, pp. 3142-3159, 2011/11/01/ 2011, doi: https://doi.org/10.1016/j.ijsolstr.2011.07.008.

[9] L.-H. Chen et al., "Soft Spherical Tensegrity Robot Design Using Rod-Centered Actuation and Control," Journal of Mechanisms and Robotics, vol. 9, no. 2, 2017, doi: $10.1115 / 1.4036014$. 
[10] S. Liu, Q. Li, P. Wang, and F. Guo, "Kinematic and static analysis of a novel tensegrity robot," Mechanism and Machine Theory, vol. 149, p. 103788, 2020/07/01/ 2020, doi: https://doi.org/10.1016/j.mechmachtheory.2020.103788.

[11] J. Zha, X. Wu, J. Kroeger, N. Perez, and M. W. Mueller, "A collision-resilient aerial vehicle with icosahedron tensegrity structure," in 2020 IEEE/RSJ International Conference on Intelligent Robots and Systems (IROS), 24 Oct.-24 Jan. 2021 2020, pp. 1407-1412, doi: 10.1109/IROS45743.2020.9341236.

[12] A. Kuzdeuov, M. Rubagotti, and H. A. Varol, "Neural Network Augmented Sensor Fusion for Pose Estimation of Tensegrity Manipulators," IEEE Sensors Journal, vol. 20, no. 7, pp. 3655-3666, 2020, doi: 10.1109/JSEN.2019.2959574.

[13] J. Shintake, D. Zappetti, T. Peter, Y. Ikemoto, and D. Floreano, "Bio-inspired Tensegrity Fish Robot," in 2020 IEEE International Conference on Robotics and Automation (ICRA), 31 May-31 Aug. 2020 2020, pp. 2887-2892, doi: 10.1109/ICRA40945.2020.9196675.

[14] R. E. Vasquez, Crane, Carl D., III, and J. C. Correa, "Analysis of a Planar Tensegrity Mechanism for Ocean Wave Energy Harvesting," Journal of Mechanisms and Robotics, vol. 6, no. 3, 2014, doi: 10.1115/1.4027703.

[15] V. Muralidharan and P. Wenger, "Optimal design and comparative study of two antagonistically actuated tensegrity joints," Mechanism and Machine Theory, vol. 159, p. 104249, 2021/05/01/ 2021, doi: https://doi.org/10.1016/j.mechmachtheory.2021.104249.

[16] N. K. Pham and E. A. Peraza Hernandez, "Modeling and Design Exploration of a Tensegrity-Based Twisting Wing," Journal of Mechanisms and Robotics, vol. 13, no. 3, 2021, doi: 10.1115/1.4050149.

[17] Y. Chen, J. Feng, and Y. Wu, "Novel Form-Finding of Tensegrity Structures Using Ant Colony Systems," Journal of Mechanisms and Robotics, vol. 4, no. 3, 2012, doi: 10.1115/1.4006656.

[18] S. Ehara and Y. Kanno, "Topology design of tensegrity structures via mixed integer programming," International Journal of Solids and Structures, vol. 47, no. 5, pp. 571-579, 2010/03/01/ 2010, doi: https://doi.org/10.1016/j.ijsolstr.2009.10.020.

[19] K. Koohestani, "Form-finding of tensegrity structures via genetic algorithm," International Journal of Solids and Structures, vol. 49, no. 5, pp. 739-747, 2012/03/01/ 2012, doi: https://doi.org/10.1016/j.ijsolstr.2011.11.015.

[20] Y. Wang, X. Xu, and Y. Luo, "Form-finding of tensegrity structures via rank minimization of force density matrix," Engineering Structures, vol. 227, p. 111419, 2021/01/15/ 2021, doi: https://doi.org/10.1016/j.engstruct.2020.111419.

[21] L.-Y. Zhang, S.-X. Zhu, S.-X. Li, and G.-K. Xu, "Analytical form-finding of tensegrities using determinant of force-density matrix," Composite Structures, vol. 189, pp. 87-98, 2018/04/01/ 2018, doi: https://doi.org/10.1016/j.compstruct.2018.01.054.

[22] Y. Chen, Q. Sun, and J. Feng, "Improved form-finding of tensegrity structures using blocks of symmetry-adapted force density matrix," Journal of Structural Engineering, vol. 144, no. 10, p. 04018174, 2018.

[23] B. Nouri Rahmat Abadi, S. M. Mehdi Shekarforoush, M. Mahzoon, and M. Farid, "Kinematic, Stiffness, and Dynamic Analyses of a Compliant Tensegrity 
Mechanism," Journal of Mechanisms and Robotics, vol. 6, no. 4, 2014, doi: 10.1115/1.4027699.

[24] R. Wang, R. Goyal, S. Chakravorty, and R. E. Skelton, "Model and Data Based Approaches to the Control of Tensegrity Robots," IEEE Robotics and Automation Letters, vol. 5, no. 3, pp. 3846-3853, 2020, doi: 10.1109/LRA.2020.2979891.

[25] M. Zhang et al., "Deep reinforcement learning for tensegrity robot locomotion," in 2017 IEEE International Conference on Robotics and Automation (ICRA), 29 May-3 June 2017 2017, pp. 634-641, doi: 10.1109/ICRA.2017.7989079.

[26] J. Luo, R. Edmunds, F. Rice, and A. M. Agogino, "Tensegrity Robot Locomotion Under Limited Sensory Inputs via Deep Reinforcement Learning," in 2018 IEEE International Conference on Robotics and Automation (ICRA), 21-25 May 2018 2018, pp. 6260-6267, doi: 10.1109/ICRA.2018.8463144.

[27] B. Cera and A. M. Agogino, "Multi-Cable Rolling Locomotion with Spherical Tensegrities Using Model Predictive Control and Deep Learning," in 2018 IEEE/RSJ International Conference on Intelligent Robots and Systems (IROS), 15 Oct. 2018 2018, pp. 1-9, doi: 10.1109/IROS.2018.8594401.

[28] S. Savin, O. Balakhnov, and A. Klimchik, "Convex Optimization-based Stiffness Control for Tensegrity Robotic Structures," in 2020 28th Mediterranean Conference on Control and Automation (MED), 15-18 Sept. 2020 2020, pp. 990995, doi: 10.1109/MED48518.2020.9182915.

[29] A. Zhakatayev, B. Abdikadirova, S. Sarmonov, and H. A. Varol, "Dynamics of Tensegrity Robots With Negative Stiffness Elements," IEEE Access, vol. 8, pp. 187114-187125, 2020, doi: 10.1109/ACCESS.2020.3031279.

[30] P. Schorr, L. Zentner, K. Zimmermann, and V. Böhm, "Jumping locomotion system based on a multistable tensegrity structure," Mechanical Systems and Signal Processing, vol. 152, p. 107384, 2021/05/01/ 2021, doi: https://doi.org/10.1016/j.ymssp.2020.107384.

[31] D. Melancon, B. Gorissen, C. J. García-Mora, C. Hoberman, and K. Bertoldi, "Multistable inflatable origami structures at the metre scale," Nature, vol. 592, no. 7855, pp. 545-550, 2021/04/01 2021, doi: 10.1038/s41586-021-03407-4.

[32] P. Sareh, P. Chermprayong, M. Emmanuelli, H. Nadeem, and M. Kovac, "Rotorigami: A rotary origami protective system for robotic rotorcraft," Science Robotics, vol. 3, no. 22, p. eaah5228, 2018, doi: 10.1126/scirobotics.aah5228.

[33] D. Dureisseix, "An Overview of Mechanisms and Patterns with Origami," International Journal of Space Structures, vol. 27, no. 1, pp. 1-14, 2012, doi: 10.1260/0266-3511.27.1.1.

[34] J. L. Rohmer, E. A. Peraza Hernandez, R. E. Skelton, D. J. Hartl, and D. C. Lagoudas, "An Experimental and Numerical Study of Shape Memory AlloyBased Tensegrity/Origami Structures," in ASME 2015 International Mechanical Engineering Congress and Exposition, 2015, vol. Volume 9: Mechanics of Solids, Structures and Fluids, V009T12A064, doi: 10.1115/imece2015-51928. [Online]. Available: https://doi.org/10.1115/IMECE2015-51928

[35] A. Klimchik, A. Pashkevich, and D. Chablat, "Fundamentals of manipulator stiffness modeling using matrix structural analysis," Mechanism and Machine Theory, vol. 133, pp. 365-394, 2019/03/01/ 2019, doi: https://doi.org/10.1016/j.mechmachtheory.2018.11.023. 
[36] D. Deblaise, X. Hernot, and P. Maurine, "A systematic analytical method for PKM stiffness matrix calculation," in IEEE International Conference on Robotics and Automation (ICRA 2006), 2006: IEEE, pp. 4213-4219.

[37] A. Klimchik, D. Chablat, and A. Pashkevich, "Stiffness modeling for perfect and non-perfect parallel manipulators under internal and external loadings," Mechanism and Machine Theory, vol. 79, pp. 1-28, 9// 2014, doi: http://dx.doi.org/10.1016/j.mechmachtheory.2014.04.002.

[38] A. Pashkevich, A. Klimchik, and D. Chablat, "Enhanced stiffness modeling of manipulators with passive joints," Mechanism and machine theory, vol. 46, no. 5, pp. 662-679, 2011. [Online]. Available: http://ac.elscdn.com/S0094114X10002338/1-s2.0-S0094114X10002338main.pdf? tid=1c9a50b0-af4a-11e3-b31e00000aacb35f\&acdnat $=1395222039$ de58abf7077cb2a2b791e10dc95b7b73.

[39] C. Quennouelle and C. á. Gosselin, "Stiffness matrix of compliant parallel mechanisms," in Advances in Robot Kinematics: Analysis and Design: Springer, 2008, pp. 331-341.

[40] H. Liu, T. Huang, D. G. Chetwynd, and A. Kecskeméthy, "Stiffness Modeling of Parallel Mechanisms at Limb and Joint/Link Levels," IEEE Transactions on Robotics, vol. 33, no. 3, pp. 734-741, 2017, doi: 10.1109/TRO.2017.2654499.

[41] A. Klimchik, D. Chablat, and A. Pashkevich, "Static stability of manipulator configuration: Influence of the external loading," European Journal of Mechanics - A/Solids, vol. 51, pp. 193-203, 5// 2015, doi: http://dx.doi.org/10.1016/j.euromechsol.2014.12.010.

[42] M. Carricato and J. Merlet, "Stability Analysis of Underconstrained Cable-Driven Parallel Robots," IEEE Transactions on Robotics, vol. 29, no. 1, pp. 288-296, 2013, doi: 10.1109/TRO.2012.2217795.

[43] M. Arsenault and C. M. Gosselin, "Kinematic, static and dynamic analysis of a planar 2-DOF tensegrity mechanism," Mechanism and Machine Theory, vol. 41, no. 9, pp. 1072-1089, 2006/09/01/ 2006, doi: https://doi.org/10.1016/j.mechmachtheory.2005.10.014.

[44] M. Furet, M. Lettl, and P. Wenger, "Kinematic analysis of planar tensegrity 2-X manipulators," in International Symposium on Advances in Robot Kinematics, 2018: Springer, pp. 153-160.

[45] P. Wenger and D. Chablat, "Kinetostatic analysis and solution classification of a class of planar tensegrity mechanisms," Robotica, vol. 37, no. 7, pp. 1214-1224, 2019, doi: 10.1017/S026357471800070X.

[46] Y. Moon, C. D. Crane, III, and R. G. Roberts, "Position and Force Analysis of a Planar Tensegrity-Based Compliant Mechanism," Journal of Mechanisms and Robotics, vol. 4, no. 1, 2012, doi: 10.1115/1.4005531.

[47] G. Ilewicz and A. Harlecki, "Multi-objective optimization and linear buckling of serial chain of a medical robot tool for soft tissue surgery," IAES International Journal of Robotics and Automation, vol. 9, no. 1, p. 17, 2020.

[48] H. Wang and A. Yamamoto, "Analyses and Solutions for the Buckling of Thin and Flexible Electrostatic Inchworm Climbing Robots," IEEE Transactions on Robotics, vol. 33, no. 4, pp. 889-900, 2017, doi: 10.1109/TRO.2017.2690302. 
[49] A. Yamada, H. Mameda, H. Mochiyama, and H. Fujimoto, "A compact jumping robot utilizing snap-through buckling with bend and twist," in 2010 IEEE/RSJ International Conference on Intelligent Robots and Systems, 18-22 Oct. 2010 2010, pp. 389-394, doi: 10.1109/IROS.2010.5652928.

[50] W. Zhao, A. Pashkevich, A. Klimchik, and D. Chablat, "Stiffness Analysis of a New Tensegrity Mechanism based on Planar Dual-triangles," in Proceedings of the 17th International Conference on Informatics in Control, Automation and Robotics, 2020, vol. 1, pp. 402-411.

[51] W. Zhao, A. Pashkevich, A. Klimchik, and D. Chablat, "The Stability and Stiffness Analysis of a Dual-Triangle Planar Rotation Mechanism," in ASME 2020 International Design Engineering Technical Conferences and Computers and Information in Engineering Conference, 2020, vol. Volume 10: 44th Mechanisms and Robotics Conference (MR), V010T10A064, doi: 10.1115/detc2020-22076. [Online]. Available: https://doi.org/10.1115/DETC2020-22076

[52] S.-F. Chen and I. Kao, "Conservative congruence transformation for joint and Cartesian stiffness matrices of robotic hands and fingers," The International Journal of Robotics Research, vol. 19, no. 9, pp. 835-847, 2000. 